\title{
Multiband coupling and nuclear softness in optical model calculations for even-even and odd- $A$ actinides
}

\author{
Dmitry Martyanov ${ }^{1, *}$, Efrem Soukhovitskiĩ ${ }^{1}$, Roberto Capote ${ }^{2, * *}$, José M. $_{\text {Quesada }}{ }^{3}$, and Satoshi Chiba ${ }^{4}$ \\ ${ }^{1}$ Joint Institute for Energy and Nuclear Research, 220109, Minsk-Sosny, Belarus \\ ${ }^{2}$ NAPC-Nuclear Data Section, International Atomic Energy Agency, Vienna A-1400, Austria \\ ${ }^{3}$ Departamento de Física Atómica, Molecular y Nuclear, Universidad de Sevilla, E-41080 Sevilla, Spain \\ ${ }^{4}$ Laboratory for Advanced Nuclear Energy, Institute of Innovative Research, Tokyo Institute of Technology, Japan
}

\begin{abstract}
A new dispersive multiband coupled channels optical model with soft-rotator "effective" deformations is proposed to describe nucleon scattering on even-even and odd- $A$ actinides. The impact of the introduction of axial and non-axial dynamical deformations that describe nuclear softness is discussed. Softness and multiband coupling are shown to change compound-nucleus formation cross section by up to $\approx 10 \%$ for incident neutron energies below $1 \mathrm{MeV}$.
\end{abstract}

\section{Introduction}

Conventional coupled-channel analysis of nucleon scattering by collective nuclei considers a target to be either vibrational or rotational [1]. In the first case small vibrational deformations of a spherical nucleus cause perturbations of an optical potential; they are expressed through deformations using Taylor expansion of the potential. The second case describes well-deformed nuclei and uses multipolar expansion of the potential where both vibrational and/or rotational states can be combined [2, 3]. Actinides feature large static deformations in the ground state combined with excited vibrational bands that start above $\approx 500-600 \mathrm{keV}$ of excitation energy in even-even actinides. Such nuclear structure could not be accommodated within existing "conventional" coupled-channel models. Moreover, rigid-rotor structure model overestimates energies of high-spin levels (e.g., difference is $\approx 10 \%$ for the $10^{+}$level of the ground state band in eveneven actinides) [4]).

Coupled-channel models using more complex nuclear structure, e.g., a Soft Rotator Model (SRM) [5, 6], have been proposed to describe nucleon scattering on spherical "soft-nuclei". While giving adequate results for structural materials, the SRM with potential decomposition around the spherical shape demonstrates poor performance for nucleon scattering on actinides. Those problems are traced to convergence problems of the potential expansion near the spherical shape for well-deformed nuclei. Therefore, new investigation was warranted.

How large is the impact of the multiple-band coupling and the account of softness of deformed nuclei on calculated cross sections for nucleon scattering on actinides? Unfortunately, almost all relevant inelastic scattering data

\footnotetext{
*e-mail: martyanov@gmail.com

**e-mail: r.capotenoy@iaea.org
}

in EXFOR [7, 8] for actinides describing neutron scattering excitation of levels from non-ground-state bands are obtained for neutron energies less than $3.5 \mathrm{MeV}$, and hence have a large component due to the compound-nucleus decay. The situation is more favorable for inelastic proton scattering experiments [9, 10] at 26 and $35 \mathrm{MeV}$. Measured (p,p') data on several nuclei including ${ }^{238} \mathrm{U}$ show considerable excitation of non-ground-state band levels, comparable with the excitation of the $6^{+}$level from the ground-state band. The energy of incident protons (26 and $35 \mathrm{MeV}$ ) allows to assign these scattering data to a direct process, described by optical model only. This implies that the multiband coupled channels model that includes excited levels from additional bands is essential for accurate optical calculations, which goes in the same direction than Dietrich et al. findings for the extended GS rotational band [11].

A dispersive coupled channels rigid-rotor optical model for deformed nuclei [12-14] was used as a starting point of new development. That potential has been shown to be approximately Lane consistent [15] and use analytically calculated dispersive contributions [16]. Then, using linear Taylor expansion of an optical potential near axially deformed shape, a generalization of the model was proposed, that allowed to account for small dynamic deformations of statically deformed nuclei [17]. Underlying rigidrotor structure of excited bands was preserved and dynamical deformations were assumed to be independent of the excited states and were taken as averaged over the whole corresponding excited band. "Effective" deformations for interband coupling were treated as adjustable parameters for both even-even and odd- $A$ nuclides, and for the later vibrational excitations were neglected. As a next step the SRM, describing low-energy collective excited states of a soft deformed even-even nucleus below $1 \mathrm{MeV}$, was used 
to compute "effective" deformations [18]. This allowed to perform more robust and reliable calculations with an account of multiband coupling and softness of target nucleus on even-even targets.

Model-driven multiband coupling for odd- $A$ nuclei and detailed analysis of main effects that arise from nucleus softness in coupled channels calculations are discussed within this work.

\section{2 "Effective" deformations}

The key quantities of this approach — "effective" deformations - are in fact matrix elements of nucleus deformation operators. They indicate how nucleus shape changes upon transition between its states. Here we consider the following dynamic deformations of a nucleus shape: nonaxial quadrupole, axial octupole, and volume conservation correction (see $[17,18])$. This results in "effective" deformations:

$$
\begin{aligned}
& {\left[\beta_{2}\right]_{e f f}=\left\langle n_{i}\left(\beta_{2}\right)\left|\delta \beta_{2}\right| \beta_{20} \mid n_{f}\left(\beta_{2}\right)\right\rangle} \\
& {\left[\beta_{3}\right]_{e f f}=\left\langle n_{i}\left(\beta_{3}\right)\left|\beta_{3}\right| \beta_{20} \mid n_{f}\left(\beta_{3}\right)\right\rangle} \\
& {\left[\gamma_{20}\right]_{e f f}=\left\langle n_{i}(\gamma)|\cos \gamma-1| n_{f}(\gamma)\right\rangle} \\
& {\left[\gamma_{22}\right]_{e f f}=\left\langle n_{i}(\gamma)|\sin \gamma| \sqrt{2} \mid n_{f}(\gamma)\right\rangle} \\
& {\left[\beta_{2}^{2}\right]_{e f f}=\left\langle n_{i}\left(\beta_{2}\right)\left|\delta \beta_{2}^{2}\right| \beta_{20}^{2} \mid n_{f}\left(\beta_{2}\right)\right\rangle} \\
& {\left[\beta_{3}^{2}\right]_{e f f}=\left\langle n_{i}\left(\beta_{3}\right)\left|\beta_{3}^{2}\right| \beta_{20}^{2} \mid n_{f}\left(\beta_{3}\right)\right\rangle} \\
& {\left[\beta_{0}\right]_{e f f}=-\frac{\beta_{20}}{\sqrt{4 \pi}}\left[2\left[\beta_{2}\right]_{e f f}+\left[\beta_{2}^{2}\right]_{e f f}+\left[\beta_{3}^{2}\right]_{e f f}\right]}
\end{aligned}
$$

here $\beta_{2}$ is the longitudinal quadrupole deformation with small vibrations $\delta \beta_{2}$ around the static deformation of ground state $\beta_{20}, \gamma$ - transverse quadrupole deformation, $\beta_{3}$ - octupole deformation, $\left|n_{i}\right\rangle$ and $\left|n_{f}\right\rangle$ are corresponding parts of the initial and final state nuclear wavefunctions describing the low-lying collective states (they are typically factorized for different types of motion). These wave-functions may depend on nuclear spin due to rotational-vibrational interaction. "Effective" deformations serve as the strength that normalize the coupled channels matrix elements: $\left[\beta_{2}\right]_{\text {eff }}$ and $\left[\gamma_{20}\right]_{\text {eff }}$ control coupling to $\beta$ - and $\gamma$-vibrational bands and also rotational stretching, $\left[\gamma_{22}\right]_{\text {eff }}$ provides coupling to anomalous vibrational band with $K=2$, and $\left[\beta_{0}\right]_{e f f}$ describes an additional coupling due to the nuclear volume conservation condition.

As discussed in Ref. [17] treating these values as fitting coefficients avoid the use of nuclear structure models beyond the rigid rotor. However, to reduce the number of model parameters, it is far better to try to derive the "effective" deformations from an structure model. This also allows to apply the derived couplings for nuclei having less experimental data for the fit. Soft-rotator model [5] is a sound structure model that can achieve good description of the low-lying collective structure of even-even actinides. It can be used to calculate the "effective" deformations given by Eq. (1), but we do not know a comparable nuclear model for odd- $A$ ones. We would like to consider not only the single-particle excitations for odd- $A$ nuclei as done in Ref. [17], but also to consider the one-phonon collective core excitations as discussed in the next Section.

\section{Approach to odd- $A$ nuclides}

Considering deformed heavy odd- $A$ nucleus as an eveneven core and an unpaired nucleon moving in the eveneven core field, we can build a plausible description of low-lying collective states [19] with wave functions partially factorized by single-particle and core dynamic variables. But perturbational account of vibrational-rotational interaction, and strong coupling of single-particle and transverse vibration/rotation of the core hinder direct application of existing formalism. On the other hand for heavy nuclei with large $A$ values the polarizing effects of the single unpaired nucleon should not affect the collective behavior of the core, in particular, nuclear softness, which is determined mainly by even-even core, and hence should vary smoothly for nearby nuclei like other collective properties, e.g., nuclear shape or deformations.

Under these considerations to calculate "effective" deformations of an odd- $A$ nucleus we use wave functions of an even-even nucleus, corresponding to a core of the odd one. In this case coupling between levels of GS, $\beta-$, and octupolar bands of and odd- $A$ nucleus can be calculated using SRM, being restricted only to bands built on the same single-particle state of the odd- $A$ nucleus. This condition is needed as it maximizes the overlap of the singleparticle wave functions of the initial and final states, being the single-particle overlap equal exactly one for the same single-particle state due to the orthogonality condition. Though many other low-lying collective bands exist for an odd- $A$ nuclei, the discussed bands are expected to have the strongest coupling, and therefore, a maximum impact on the calculated optical-model cross sections.

The last missing piece in this scheme is the mapping of the odd- $A$ nucleus states onto the corresponding eveneven ("core") states needed to calculate the coupling matrix elements. While vibrational quantum numbers can be used straightforwardly, spins require additional explanations. First, GS spin of an odd- $A$ nucleus is determined by the full angular momentum of the corresponding singleparticle state as the even-even core spin is zero. Hence the nucleus rotation (sequence of states along the rotational band) can be attributed to the core. Second, each rotational band of an odd- $A$ nucleus in fact consists of two sub-bands (one with the lowest state and its satellite) with spin increment equal to 2 . As a result, a typical even-even nucleus spin sequence is assigned to every sub-band of an odd- $A$ nucleus. Example of such assignment can be seen in Table 1.

\section{Calculation algorithm}

Calculations were performed using the following scheme. First, recommended collective-level energies of even-even targets (assumed to be a core of an odd- $A$ target) were retrieved from the ENSDF database [20] and used to fit 
Table 1. Coupling scheme for ${ }^{233} \mathrm{U}$ and ${ }^{238} \mathrm{U}$.

\begin{tabular}{|c|c|c|c|c|}
\hline \multicolumn{3}{|c|}{${ }^{233} \mathrm{U}$} & \multicolumn{2}{|c|}{${ }^{238} \mathrm{U}$} \\
\hline$I_{\text {core }}^{\pi}$ & $I^{\pi}$ & $E, \mathrm{MeV}$ & $I^{\pi}$ & $E, \mathrm{MeV}$ \\
\hline \multicolumn{3}{|c|}{ GS band } & \multicolumn{2}{|c|}{ GS band } \\
\hline $0^{+}$ & $5 / 2^{+}$ & 0.000 & $0^{+}$ & 0.000 \\
\hline $2^{+}$ & $9 / 2^{+}$ & 0.092 & $2^{+}$ & 0.045 \\
\hline $4^{+}$ & $13 / 2^{+}$ & 0.229 & $4^{+}$ & 0.148 \\
\hline $6^{+}$ & $17 / 2^{+}$ & 0.411 & $6^{+}$ & 0.307 \\
\hline $8^{+}$ & $21 / 2^{+}$ & 0.635 & $8^{+}$ & 0.518 \\
\hline \multicolumn{3}{|c|}{ GS band satellite } & $10^{+}$ & 0.776 \\
\hline $0^{+}$ & $7 / 2^{+}$ & 0.040 & $12^{+}$ & 1.077 \\
\hline $2^{+}$ & $11 / 2^{+}$ & 0.155 & \multicolumn{2}{|c|}{ Non-axial band } \\
\hline $4^{+}$ & $15 / 2^{+}$ & 0.315 & $2^{+}$ & 1.060 \\
\hline $6^{+}$ & $19 / 2^{+}$ & 0.518 & $3^{+}$ & 1.106 \\
\hline $8^{+}$ & $23 / 2^{+}$ & 0.762 & $4^{+}$ & 1.163 \\
\hline \multicolumn{3}{|c|}{ Octupole band } & \multicolumn{2}{|c|}{ Octupole band } \\
\hline $1^{-}$ & $5 / 2^{-}$ & 0.749 & $1^{-}$ & 0.680 \\
\hline $3^{-}$ & $9 / 2^{-}$ & 0.838 & $3^{-}$ & 0.732 \\
\hline \multicolumn{3}{|c|}{ Octupole band satellite } & $5^{-}$ & 0.827 \\
\hline $1^{-}$ & $7 / 2^{-}$ & 0.790 & $7^{-}$ & 0.966 \\
\hline $3^{-}$ & $11 / 2^{-}$ & 0.916 & $9^{-}$ & 1.151 \\
\hline \multicolumn{3}{|c|}{$\beta$-vibration band } & \multicolumn{2}{|c|}{$\beta$-vibration band } \\
\hline $0^{+}$ & $5 / 2^{+}$ & 1.311 & $0^{+}$ & 0.993 \\
\hline \multicolumn{3}{|c|}{$\beta$ - band satellite } & $2^{+}$ & 1.037 \\
\hline \multirow[t]{6}{*}{$0^{+}$} & \multirow[t]{6}{*}{$7 / 2^{+}$} & \multirow[t]{6}{*}{1.347} & $4^{+}$ & 1.131 \\
\hline & & & $6^{+}$ & 1.269 \\
\hline & & & \multicolumn{2}{|c|}{$\gamma$-vibration band } \\
\hline & & & $0^{+}$ & 0.927 \\
\hline & & & $2^{+}$ & 0.966 \\
\hline & & & $4^{+}$ & 1.056 \\
\hline
\end{tabular}

the SRM parameters for each nuclide. Then, all necessary "effective" deformations were calculated using the fitted SRM model. Experimental data on nucleon scattering on ${ }^{238} \mathrm{U}$ and ${ }^{232} \mathrm{Th}$ targets allowed to build a regional optical potential model, and fit the static deformation parameters. For the considered odd- $A$ nucleus, the scant data available allowed to fit the static deformations only, using the same potential derived from ${ }^{238} \mathrm{U}$ and ${ }^{232} \mathrm{Th}$ scattering. Description of experimental data is similar to what was achieved in Ref. [17] including the total cross section ratio for a pair of nuclides. However, current model has a lower number of parameters and more accurately predict scattering cross sections on odd- $A$ targets. Calculations of nucleon induced reactions on ${ }^{238} \mathrm{U}$ and ${ }^{233} \mathrm{U}$ targets are used as examples to analyze and compare the changes due to the account of nuclear softness in optical model calculations. The coupling schemes and assigned core spins for ${ }^{233} \mathrm{U}$ are shown in Table $1 .{ }^{233} \mathrm{U}$ is chosen since ENSDF database for it has a maximum number of ready-to-use rotational bands clearly assigned to vibrational collective excitations of the core built on the same single-particle state as the GS band.

\section{Results}

Results are presented for the compound nucleus $(\mathrm{CN})$ formation cross sections as this is a very sensitive quantity,
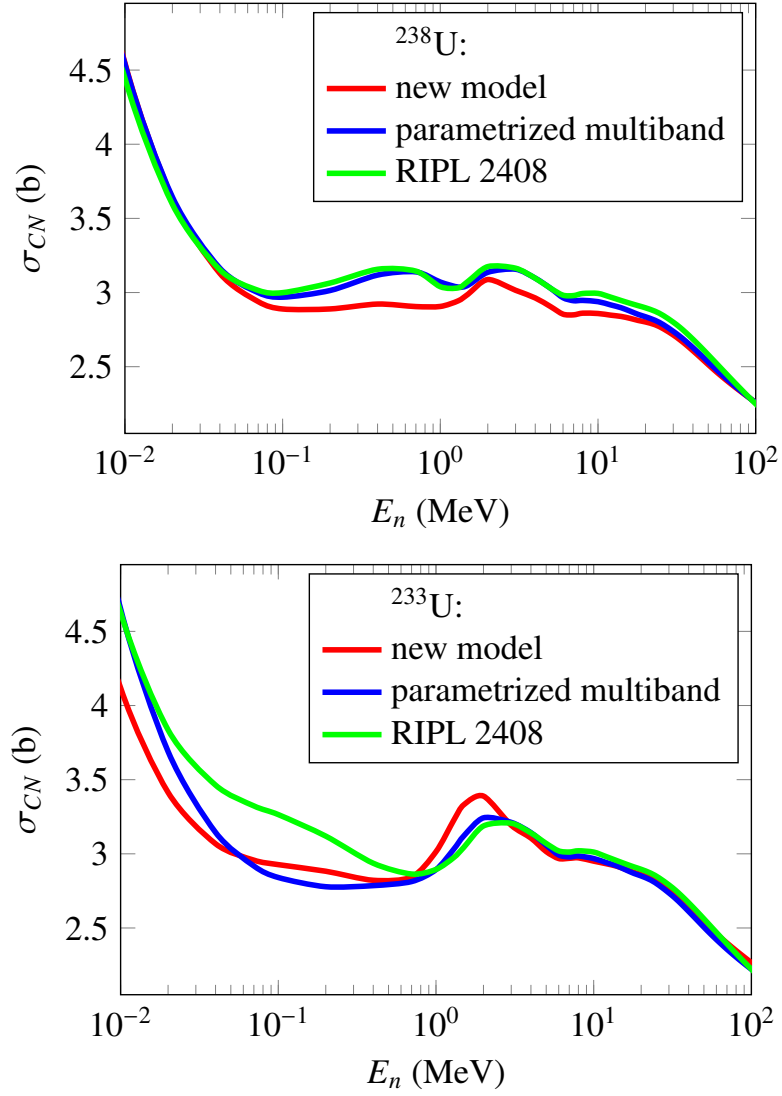

Figure 1. Calculated $\mathrm{CN}$ formation cross sections for neutron scattering on ${ }^{238} \mathrm{U}$ (top) and ${ }^{233} \mathrm{U}$ (bottom) using three Laneconsistent dispersive optical models: RIPL 2408 - rigid-rotor GS band coupling [12-14], multiband coupling with fitted "effective" deformations [17], and current model with SRM-derived multiband coupling.

which is directly linked to needed nuclear transmission coefficients for calculations of the compound-nucleus decay using the Hauser-Feshbach theory [21]. Most of results are shown as a symmetrized ratio of $\mathrm{CN}$ formation cross sections: $R(A ; B)=\frac{\sigma_{C N}^{A}-\sigma_{C N}^{B}}{\sigma_{C N}^{A}+\sigma_{C N}^{B}}$.

How calculated $\mathrm{CN}$ formation cross sections change upon the development of Lane-consistent dispersive coupled channels optical model? Fig. 1 compare predictions of 3 phenomenological optical model potentials fitted to the same experimental data: a rigid rotor potential RIPL 2408 [12-14] ${ }^{1}$ with 5 (for ${ }^{238} \mathrm{U}$ ) or 6 (for ${ }^{233} \mathrm{U}$ ) levels from the GS band in the coupling scheme; a multiband coupling potential from [17] with 20 levels from 5 bands (for ${ }^{238} \mathrm{U}$ ) or 16 levels from 3 bands (for ${ }^{233} \mathrm{U}$ ) that uses effective deformation as fitting parameters, and finally a potential developed in this work that considers nuclear softness, SRM derived "effective" deformations, and slightly larger coupling scheme. $\mathrm{CN}$ cross section predictions vary up to $300 \mathrm{mb}$ (about 10\%) for different models mainly below $1 \mathrm{MeV}$.

Derived nonelastic cross sections for neutron scattering on ${ }^{238} U$ target using the three different optical models discussed above are compared in Fig. 2 with available experimental data in EXFOR [22-26] as well as with es-

\footnotetext{
${ }^{1}$ referred in this work as the RIPL 2408 potential by using the keyword from the RIPL optical-model database [28].
} 


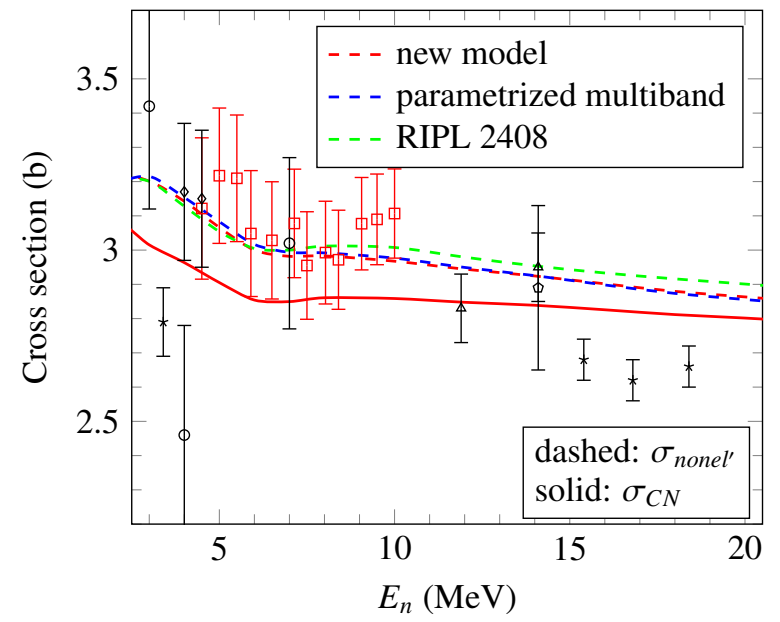

Figure 2. Calculated nonelastic cross sections (dashed) for neutron scattering on ${ }^{238} \mathrm{U}$ using three dispersive optical model potentials: RIPL 2408 - rigid-rotor GS band coupling [12-14], multiband coupling with fitted "effective" deformations [17], and current model with SRM-derived multiband coupling. Derived experimental nonelastic neutron scattering cross sections from EXFOR [22-26] (black points) and Dietrich [27] (red points) are compared to calculations. Note that the direct inelastic cross sections of the first two excited levels were also included as quasielastic cross sections to derive the plotted non-elastic cross section $\sigma_{\text {nonel }}$. Calculated $\mathrm{CN}$ formation cross section using the new SRM model is shown as solid line for comparison.

timated non-elastic cross sections by Dietrich $[27]^{2}$. An acceptable agreement is seen between calculated (dashed lines) and experimental cross sections (black and red points); these data were not used in the potential fitting. Note that nonelastic data should be compared with optical model calculations above $3 \mathrm{MeV}$ to avoid the impact of the compound elastic cross section on the experimental data. Experimental resolution above $3 \mathrm{MeV}$ does not allow separating the first two inelastic levels from the elastic one. Therefore, the calculated nonelastic is derived as the total minus the direct elastic and inelastic scattering cross section of the first two excited levels located at 45 and $149 \mathrm{keV}$. In the same plot the calculated compound nucleus cross section (solid line) is shown using the potential proposed in this work; this cross section is lower than the measured nonelastic one as the inelastic scattering to additional coupled levels is non-negligible.

What is the impact of the consideration of the nuclear softness?

\subsection{Multiband coupling}

Direct level excitation cross section shown on fig. 3 demonstrates the contribution to calculated cross sections of the coupling of non-GS-band levels. Here "band" excitation cross section stands for the sum of level excitations over corresponding band. Direct non-GS-band excitation cross sections reach up to $80 \mathrm{mb}\left(60 \mathrm{mb}\right.$ for $\left.{ }^{233} \mathrm{U}\right)$ and are comparable with the direct-excitation cross sections of

\footnotetext{
${ }^{2}$ Dietrich developed a special method to obtain non-elastic cross sections [29, 30] based on the optical theorem, and the measured total cross sections and corresponding elastic angular distributions.
}

the second or third level from the GS band. This result is also qualitatively in agreement with (p,p') experimental works $[9,10]$.

Other way of analyzing the impact of non-GS-bands is to estimate the changes in $\mathrm{CN}$ formation cross section upon removal of band levels from the coupling scheme with the fixed optical potential. Fig. 4 shows the results of such calculation. Truncation of the coupling scheme leads to a significant change of $\mathrm{CN}$ formation cross section (typically up to $10 \%$ ) for incident energies lower than several $\mathrm{MeV}$ with the greatest influence of levels from $\beta$ vibration rotational band. However, this effect is probably overestimated as compensation and redistribution of inelastic cross sections occur if we refit the experimental data with the reduced coupling scheme.

\subsection{Nucleus stretching}

Nuclear softness also describes the stretching of a nucleus due to centrifugal forces that lead to the reduction of the energy of the high-spin levels. This property allows a much better description of the experimental energies of the GS band using a SRM, and is expected to have an impact on calculated cross sections. Only GS band levels were left in the coupling scheme and volume conservation correction was disabled to evaluate this effect alone. Fig. 5 shows the difference in calculated $\mathrm{CN}$ formation cross section upon enabling the stretching effect. It reaches more than $10 \%$ for projectile energy under few $\mathrm{MeV}$, highlighting the importance of nuclear stretching even when only GS band levels are coupled. This effect could not be considered in Ref. [17], as all rotational-vibrational bands in that work were assumed to be rigid. Note that softness leads to non-conservation of the projection of the angular momentum on the deformation axis (quantum number $K$ ). Therefore, the wave function of SRM includes mixture in the quantum number $K$ as derived in Ref. [17], but not used in cross-section calculations there.

\subsection{Volume conservation}

Nuclear volume conservation correction can be enforced using nuclear softness by introducing a dynamical monopolar deformation. Fig. 6 demonstrates introduced changes in calculated $\mathrm{CN}$ formation cross section. The significance of this effect is clearly seen, however calculated changes are partially caused by changes in the octupole deformation of a nucleus, so real dynamic (caused by softness) effect is lower than shown on fig. 6 .

\section{Conclusions}

The proposed application of Lane-consistent dispersive multiband coupled channels optical model with SRM "effective" deformations to odd- $A$ nuclei may potentially allow building a unified regional optical potential with multi-band coupling for both even-even and odd- $A$ actinides. Such potential will couple multiple rotationalvibrational bands and assume that actinides are statically 

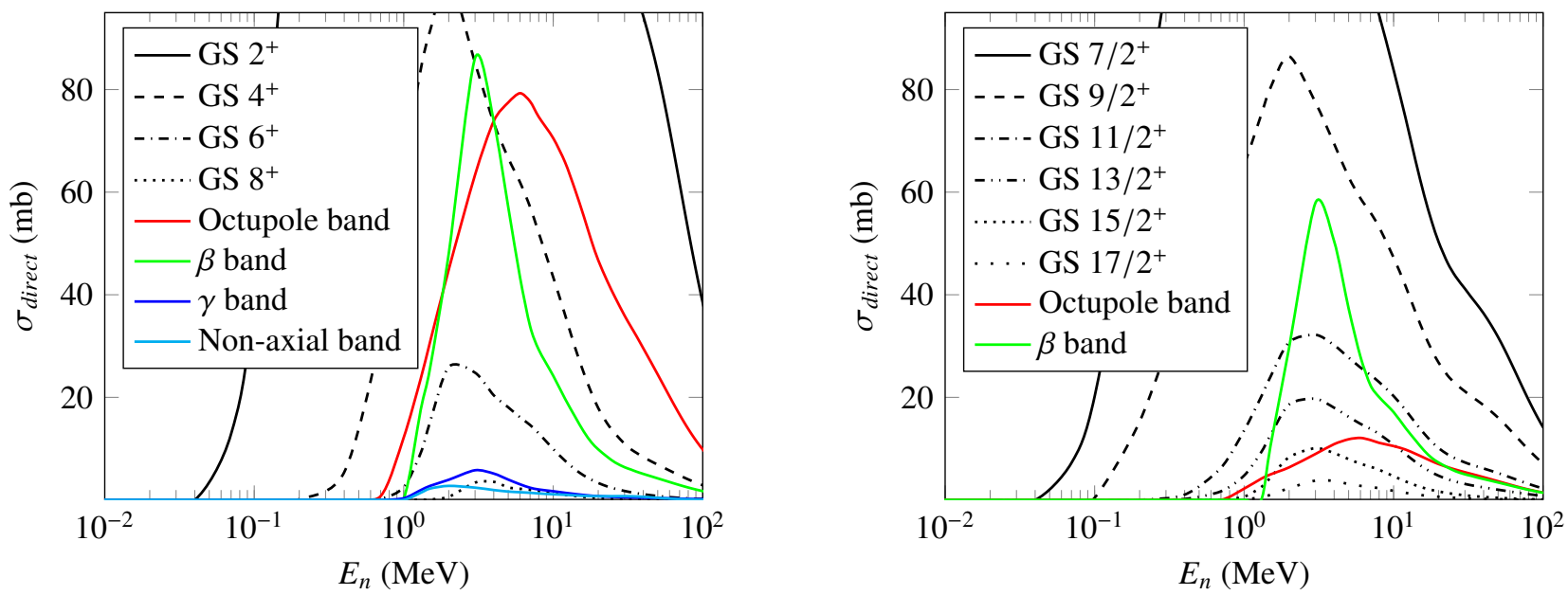

Figure 3. Direct level excitation cross sections within current model. Cross sections for levels from non-GS bands are summed over each band. Several GS band levels are shown for comparison. Left $-{ }^{238} \mathrm{U}$, right $-{ }^{233} \mathrm{U}$.
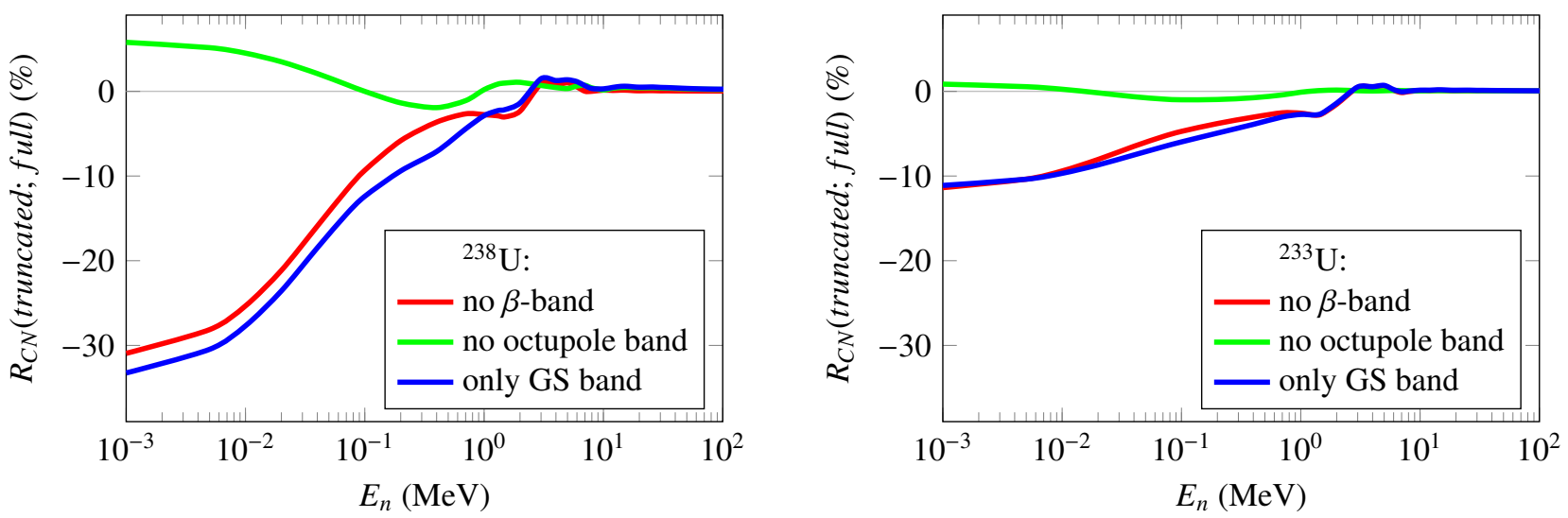

Figure 4. Change of $\mathrm{CN}$ formation cross section due to truncation of the full coupling scheme: some or all non-GS bands are removed.

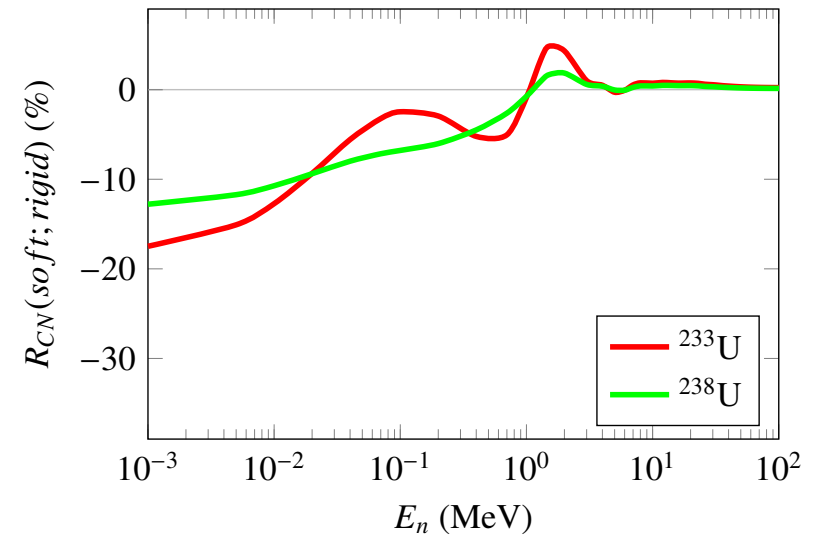

Figure 5. Change of $\mathrm{CN}$ formation cross section of axial rigidrotor model with GS band coupling due to introduction of nucleus stretching (assuming SRM-based "effective" deformations for GS band).

well-deformed nuclei which are soft to axial and non-axial dynamical vibrations.

Three phenomenological optical model potentials fitted to almost the same experimental data, but with differ-

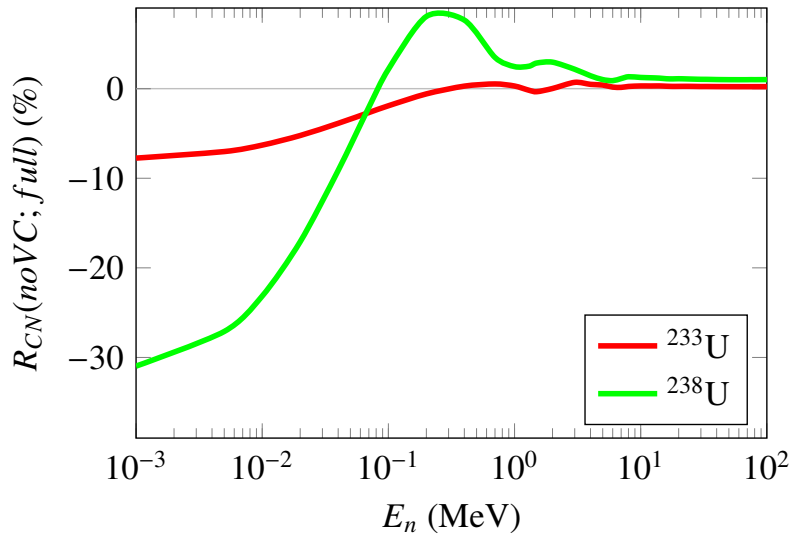

Figure 6. Change of $\mathrm{CN}$ formation cross section if the volume conservation is neglected within current model with full coupling.

ent coupling schemes and nuclear structure information, give prediction of the compound nucleus formation cross sections. These calculated $\mathrm{CN}$ formation cross sections show an spread of up to 300 millibarns (about 10\%) for ${ }^{238} \mathrm{U}$ and ${ }^{233} \mathrm{U}$ below $1 \mathrm{MeV}$. 
The impact of the introduction of the SRM description on calculated cross sections on both even-even and odd$A$ actinides was analyzed on examples of ${ }^{238} U$ and ${ }^{233} U$ targets. The effect of multiband coupling, centrifugal nucleus stretching, and volume conservation are shown to be important for accurate $\mathrm{CN}$ formation cross section calculations, with smaller impact for odd- $A$ nuclei as intuitively expected. Coupling to the vibrational $\beta$-band appears to have the largest influence on the results. Direct excitation cross sections of non-GS bands are comparable to the inelastic scattering cross section of the second or third level of the GS band.

Fitting of data for other odd- $A$ actinides requires thorough analysis of their low-lying level structure that goes beyond the information currently available in the ENDSF database [20, 31, 32]. An alternative approach may involve the use of SRM and developed local systematics of SRM parameters to estimate the energy of expected collective bands needed for the coupled-channel optical model description.

This work is partially supported by IAEA Contract 19263 and the contract with the Spanish Ministry of Science, Innovation and Universities RTI2018-098117-B-C21. DM is grateful to IAEA for travel funding that allowed to attend the conference.

\section{References}

[1] T. Tamura, Rev. Mod. Phys. 37, 679 (1965)

[2] J. Raynal, Tech. Rep. IAEA SMR-9/8, IAEA, Vienna (1970)

[3] J. Raynal, Notes on ECIS95 (1995)

[4] J.M. Quesada, D. Martyanov, E. Soukhovitskiñ, R. Capote, S. Chiba, EPJ Web Conf. 146, 12013 (2017)

[5] Y.V. Porodzinskiĩ, E.S. Sukhovitskiĩ, Sov. Journ. of Nucl. Phys. 53, 64 (1991)

[6] Y.V. Porodzinskiũ, E.S. Sukhovitskiĩ, Phys. Atom. Nucl. 59, 228 (1996)

[7] N. Otuka, E. Dupont, V. Semkova, B. Pritychenko, A.I. Blokhin, M. Aikawa, S. Babykina, M. Bossant, G. Chen, S. Dunaeva et al., Nucl. Data Sheets (2014)

[8] Web database retrieval system for Experimental $\mathrm{Nu}$ clear Reaction Data (EXFOR). Data available online (e.g., at http://www-nds.iaea.org/exfor/)

[9] L.F. Hansen, I.D. Proctor, D.W. Heikkinen, V.A. Madsen, Phys. Rev. C 25, 189 (1982)

[10] C.H. King, J.E. Finck, G.M. Crawley, J.A. Nolen, R.M. Ronningen, Phys. Rev. C 20, 2084 (1979)

[11] F.S. Dietrich, I.J. Thompson, T. Kawano, Phys. Rev. C 85, 044611 (2012)

[12] R. Capote, E.S. Soukhovitskii, J.M. Quesada, S. Chiba, Phys. Rev. C 72, 064610 (2005)
[13] E.S. Soukhovitskii, R. Capote, J.M. Quesada, S. Chiba, Phys. Rev. C 72, 024604 (2005)

[14] R. Capote, S. Chiba, E.S. Soukhovitskiĩ, J.M. Quesada, E. Bauge, J. Nucl. Sci. Technol. 45, 333 (2008)

[15] J.M. Quesada, R. Capote, E.S. Soukhovitskii, S. Chiba, Phys. Rev. C 76, 057602 (2007)

[16] J.M. Quesada, R. Capote, A. Molina, M. Lozano, J. Raynal, Phys. Rev. C 67, 067601 (2003)

[17] E.S. Soukhovitskiĩ, R. Capote, J.M. Quesada, S. Chiba, D.S. Martyanov, Phys. Rev. C 94, 064605 (2016)

[18] D. Martyanov, E. Soukhovitskii, R. Capote, J.M. Quesada, S. Chiba, EPJ Web Conf. 146, 12031 (2017)

[19] J.M. Eisenberg, W. Greiner, Nuclear Theory, Nuclear Models (North-Holland, 1987), ISBN 9780444870735

[20] Evaluated Nuclear Structure Data File (ENSDF). Available online at https://www.nndc.bnl.gov/ensdf/, $B N L$, USA. Maintained by the international network of Nuclear Structure and Decay Data Evaluators (NSDD) at https://www-nds.iaea.org/nsdd/

[21] W. Hauser, H. Feshbach, Phys. Rev. 87, 366 (1952)

[22] R. Batchelor, W. Gilboy, J. Towle, Nucl. Phys. 65, 236 (1965)

[23] H. Bethe, J. Beyster, R. Carter, R. Henkel, R. Nobles, Tech. Rep. 1939, Los Alamos Scientific Lab, Los Alamos (1955)

[24] J. Degtjarev, V. Nadtochij, Atomnaya Energiya 11, 397 (1961)

[25] M.H. MacGregor, R. Booth, W.P. Ball, Phys. Rev. 130, 1471 (1963)

[26] J.J. Voignier, Tech. Rep. CEA-R-3503, CEA, Limeil, France (1968)

[27] F.S. Dietrich, private communication (2008)

[28] R. Capote, M. Herman, P. Obložinský, P.G. Young, S. Goriely, T. Belgya, A.V. Ignatyuk, A.J. Koning, S. Hilaire, V.A. Plujko et al., Nucl. Data Sheets 110, 3107 (2009)

[29] F.S. Dietrich, J.D. Anderson, R.W. Bauer, S.M. Grimes, Phys. Rev. C 68, 064608 (2003)

[30] F.S. Dietrich, J.D. Anderson, R.W. Bauer, S.M. Grimes, D.P. McNabb, Nucl. Sci. Eng. 159, 213 (2008)

[31] LiveChart of Nuclides, IAEA nuclear structure and decay data retrieval code, https: //www-nds. iaea.org/relnsd/vcharthtml/ VChartHTML . html

[32] National Nuclear Data Center, the NuDat 2 nuclear structure and decay database, https: //WwW . nndc . bnl.gov/nudat2/ 